\title{
RELACIÓN ENTRE HABILIDADES DE PROCESAMIENTO DE LA INFORMACIÓN Y RENDIMIENTO ACADÉMICO
}

\author{
Manuel Miljánovich C. ${ }^{*}$ Félix Quesada C., María Atalaya P., Rosa Huerta R., Jaime Aliaga T., \\ Desiderio Evangelista H.
}

\section{RESUMEN}

Los coeficientes de correlación calculados entre los puntajes totales y parciales en una Prueba de Habilidades de Procesamiento Elaborativo de la Información y los promedios de notas correspondientes a cuatro semestres académicos de una muestra de estudiantes de diversas facultades de la Universidad Nacional Mayor de San Marcos permiten sostener, como confirmación de la hipótesis planteada, que tales habilidades poseen un apreciable poder predictivo del éxito en los estudios universitarios, especialmente en las áreas de humanidades.

El estudio incluye una comparación entre la referida correlación y la hallada, en la misma muestra, entre puntajes en la Prueba de Admisión a la universidad (2003) y los promedios de notas. El resultado muestra, en general, que la Prueba de Procesamiento de la Información posee mayor poder predictivo que la Prueba de Admisión.

Palabras clave: Habilidades, procesamiento, información, predicción rendimiento académico, universidad.

\begin{abstract}
The correlative coefficients evaluated between the total and partial scores on an elaborative processing of information skills test and the averages notes corresponding to four academic semesters from a students sample of San Marcos University's diverse faculties, allow us to sustain, according to the hypotesis stated, that those skills have an apreciable predictive power of succes in university studies, specially in humanity area.

The survey included a comparison betwen the reported correlation and the located, on the same sample, between scores on the admision university test and the averages notes. The result shows in general, that the prosecution of the information test, have a larger predictive power than the admision test.
\end{abstract}

Keywords: Skills, prosecution, information, prediction yield scholastic, university.

* Psicólogo y Doctor en Educación, Catedrático Principal de la UNMSM y asesor de la Oficina Central de Admisión. 


\section{INTRODUCCIÓN}

El presente estudio forma parte de un programa de investigación cuyo tema central es la construcción y validación de una Prueba de procesamiento elaborativo de la Información, de inspiración cognitivista, como contribución al acervo de instrumentos de selección para el ingreso a universidades y propósitos afines.

La prueba en referencia fue construida y validada, en lo que atañe a validez de contenido y aspectos formales, en el año 2001 (Proyecto $N^{\circ} 1801081$ del Instituto de Investigaciones Psicológicas de la Facultad de Psicología de la Universidad Nacional Mayor de San Marcos).

El presente artículo presenta los resultados del estudio longitudinal o de seguimiento que cubrió cuatro semestres académicos, realizado con una muestra de estudiantes de diversas facultades que ingresaron en 2003, y que estuvo orientado a establecer la validez predictiva de la Prueba de Procesamiento de la Información en referencia. Este estudio constituye la segunda etapa del programa de investigación mencionado (Proyecto $\mathrm{N}^{\circ} 031801041$ ).

El problema a investigar se formuló en los siguientes términos: ¿Cuál es el grado de correlación entre las habilidades de procesamiento elaborativo de la información y el Rendimiento académico en una muestra de estudiantes pertenecientes a diversas facultades de la Universidad Nacional Mayor de San Marcos en el periodo 2003 y 2004? El aspecto pragmático de este problema puede ser traducido mediante la siguiente pregunta ¿En qué medida la prueba en referencia tiene poder predictivo respecto al éxito o fracaso en los estudios universitarios?

Según el marco teórico en el que se inscribe la presente investigación, el procesamiento de la información es un encadenamiento de actos que está siempre presente en los aprendizajes de tipo académico, en la resolución de problemas y en la ejecución de tareas propias del ámbito universitario. En efecto, la información que proviene de la observación, de la interacción con el docente, de la lectura, del estudio, de la experimentación o aquella que surge como producto de la reflexión constituye un conjunto de contenidos que se clasifican, organizan o reorganizan según las demandas de la circunstancia, los esquemas mentales y el estilo del sujeto.

Este procesamiento involucra las capacidades de discernimiento de niveles de generalidad, secuencias deductivas, secuencias cronológicas, relaciones causales, relaciones argumentativas de fundamentación y ángulos de enfoque.

Desde el punto de vista de las teorías modélicas de la memoria, que se basan en un símil funcional entre el cerebro humano y la computadora, el procesamiento reseñado puede entenderse como un esfuerzo recurrentemente enriquecido de codificación de significados y de su almacenamiento en la memoria de largo plazo, con adecuadas claves de recuperación.

El término elaborativo pone de relieve la conceptualización de creciente complejidad, la tendencia hacia lo novedoso y el ingrediente idiosincrático que caracterizan el procesamiento de la información en el plano académico, por oposición al procesamiento rutinario o meramente reproductivo.

Las hipótesis que guiaron la investigación de la que aquí damos cuenta sostienen lo siguiente:

a) Existe un grado significativo y apreciable de correlación entre los puntajes obtenidos por los estudiantes que integran la muestra en la Prueba de Procesamiento Elaborativo de la 
Información y sus promedios de notas en las asignaturas cursadas en los cuatro primeros semestres académicos de su formación profesional (Rendimiento Académico); b) La correlación entre los puntajes en la Prueba de Procesamiento Elaborativo de la Información y el Rendimiento Académico es de mayor magnitud que la correlación entre los puntajes en la Prueba de Admisión y el Rendimiento Académico.

\section{MATERIALES Y MÉTODOS}

La Prueba de Procesamiento Elaborativo de la Información fue construida y validada durante los años 2000 y 2001 (Proyecto $\mathrm{N}^{\circ} 1801081$ ) en lo que respecta a validez de contenido y aspectos formales. Mediante el estudio longitudinal realizado en el período comprendido entre los años 2003 y 2004 se pretende establecer su validez predictiva.

La prueba en mención comprende dos partes. La primera, denominada Subprueba de Selección Argumentativa, consta de 31 párrafos cortos que se refieren a diversos aspectos de un tema de relevancia mundial y de gran difusión. El sujeto debe leer y releer atenta, analítica e interpretativamente dichos párrafos para poder responder a cinco ítemes que interrogan sobre las relaciones entre los contenidos de tales párrafos. La segunda parte, denominada Subprueba de Pensamiento Estratégico, consta de cinco ítemes que plantean situaciones problemáticas de carácter hipotético que el sujeto debe analizar y evaluar poniendo en juego su capacidad de pensamiento lógico y su sentido de planificación.

Cada ítem está compuesto por un enunciado y cuatro alternativas, entre las cuales sólo una es la clave o respuesta correcta.

La confiabilidad de la prueba, la calibración de los ítemes, la evaluación del funcionamiento de las alternativas y demás propiedades psicométricas del instrumento fueron determinadas en la primera etapa del programa de investigación y algunas de ellas fueron afinadas en el estudio longitudinal o de seguimiento.

El procesamiento estadístico se efectuó fundamentalmente en base al coeficiente de correlación producto - momento de Pearson y sus correspondientes grados de significación. Previamente se determinaron los estadísticos descriptivos necesarios para comprobar el grado de aproximación de la distribución de los datos a la curva normal y las respectivas medidas de la tendencia central y de dispersión.

\section{RESULTADOS}

Lo primero que se advierte en los cuadros que presentan el producto del procesamiento estadístico de los datos es la marcada heterogeneidad de los coeficientes de correlación entre el Rendimiento Académico, expresado en los promedios ponderados de las notas de asignaturas, por una parte, y, por la otra, los puntajes totales en la Prueba de Procesamiento de la Información y los puntajes en sus subpruebas componentes o en la Prueba de Admisión 2003 y sus subpruebas, según sea el caso.

Tal heterogeneidad se extiende desde coeficientes positivos ( $r=0.319$ en la EAP de Administración, entre el Rendimiento Académico y la subprueba de Pensamiento Estratégico, coeficiente significativo al nivel del 0.01 , bilateral ), hasta coeficientes negativos e igualmente significativos ( $r=-0.163$ en la EAP de Farmacia y Bioquímica, entre el Rendimiento 
Académico y la Subprueba de Selección Argumentativa), variando en función de las carreras o escuelas académico profesionales y de las pruebas o subpruebas de las que se trate.

Teniendo en cuenta que uno de los propósitos de la prueba en referencia es servir como un instrumento de selección para el ingreso a la universidad, a manera de alternativa de los modelos en uso, no sólo se correlacionó el Rendimiento Académico con los puntajes totales en la referida Prueba de Procesamiento de la Información y sus subpruebas componentes, sino también se efectuó similar correlación, a fin de establecer comparaciones, con la Prueba de Admisión 2003 y sus subpruebas.

Los resultados muestran que hay una gran heterogeneidad en los coeficientes de correlación, en función de las pruebas y subpruebas y de las carreras o escuelas académico profesionales. Sin embargo, se perfila la tendencia de que la Prueba de Procesamiento de la Información, y especialmente su Subprueba de Pensamiento Estratégico, muestra una validez predictiva del éxito en los estudios universitarios mayor que la que presenta la Prueba de Admisión 2003 en carreras humanísticas o cercanas a ellas, mientras que la Prueba de Admisión, y especialmente su Subprueba de Conocimientos, poseen mayor validez predictiva en las carreras de ciencias e ingenierías.

\section{RENDIMIENTO ACADÉMICO VS PRUEBA DE PROCESAMIENTO DE LA INFORMACIÓN}

1.1. Lo primero que se advierte al examinar el cuadro $\mathrm{N}^{\circ} 3$ es la marcada heterogeneidad de las correlaciones, tanto en la muestra total como a nivel de las EAP. En efecto, los coeficientes de correlación varían desde $r=0.319$, entre Rendimiento Académico y la Subprueba de Pensamiento Estratégico en la EAP de Administración, hasta una correlación negativa de $r=-0.163$ entre Rendimiento Académico y la Subprueba de Selección Argumentativa en las EAP de Farmacia y Bioquímica y en la de Química.

1.2. En la muestra total, los coeficientes de correlación son significativos al nivel del 0.01 , bilateral.

1.3. En la muestra total, las correlaciones presentan el siguiente gradiente: a) Rendimiento Académico vs puntaje total en la Prueba de Procesamiento de la Información: $\mathrm{r}=$ 0.243 ; b) Rendimiento Académico vs. Subprueba de Pensamiento Estratégico: $r=$ 0.188 ; c) Rendimiento Académico vs. Subprueba de Selección Argumentativa: $r=$ 0.176 .

1.4. A nivel de las carreras o EAP, las correlaciones más altas las encontramos en: a) Administración, entre Rendimiento Académico y la Subprueba de Pensamiento Estratégico: $\mathrm{r}=0.319$, significativa al 0.01 , bilateral y entre Rendimiento Académico y el puntaje total en la Prueba de Procesamiento de la Información: $r=0.286$; b) Lingüística, entre Rendimiento Académico y la Subprueba de Pensamiento Estratégico: $r=0.243$, significativa al 0.01 , bilateral y entre Rendimiento Académico y el puntaje total en la Prueba de Procesamiento de la Información: $r=0.224$, significativa al 0.01, bilateral; c) Psicología, entre Rendimiento Académico y el puntaje total en la Prueba de Procesamiento de la Información: $r=0.178$; entre Rendimiento Académico y la Subprueba de Pensamiento Estratégico: $r=0.155$ y entre Rendimiento Académico y la Subprueba de Selección Argumentativa: $r=0.114$; d) Obstetricia, entre 
Rendimiento Académico y el puntaje total en la Prueba de Procesamiento de la Información: $r=0.134$ y entre Rendimiento Académico y la Subprueba de Pensamiento Estratégico: $r=0.133$.

1.5. A nivel de EAP, las correlaciones más bajas e incluso negativas las encontramos en:

a) Farmacia y Bioquímica: Entre Rendimiento Académico y la Subprueba de Selección Argumentativa: $r=-0.163$; entre Rendimiento Académico y el puntaje total en la Prueba de Procesamiento de la Información: $r=-0.084$ y entre Rendimiento Académico y la Subprueba de Pensamiento Estratégico: $\mathrm{r}=0.026$.

b) Física: Entre rendimiento académico y la Subprueba de Selección Argumentativa: $\mathrm{r}=-0.111$; entre Rendimiento Académico y el puntaje total en la Prueba de Procesamiento de la Información: $r=-0.028$ y entre Rendimiento Académico y la Subprueba de Pensamiento Estratégico: $r=0.068$.

c) Valores similares se hallaron en Ingeniería de Sistemas y en Química, sin embargo, en ésta última la correlación entre rendimiento académico y la Subprueba de Pensamiento Estratégico, es positiva y de apreciable cuantía: $r=0.258$.

2. CORRELACIONES ENTRE RENDIMIENTO ACADÉMICO Y LA PRUEBA DE ADMISIÓN 2003 A LA UNMSM

2.1. Las correlaciones entre rendimiento académico y la Prueba de Admisión 2003 a la UNMSM y sus subpruebas son también bastantes heterogéneas. Varían desde $r=0.398$ en Física, entre rendimiento académico y el puntaje total en la referida prueba hasta $\mathrm{r}=-0.264$ en Obstetricia, entre Rendimiento Académico y la Subprueba de Aptitud Académica.

2.2. La mayoría de las coeficientes de correlación son significativos al 0.05 y algunos al 0.01 , bilateral.

2.3. En la muestra total, las correlaciones presentan el siguiente gradiente: a) Rendimiento Académico y la Subprueba de Conocimientos: $r=0.242$; b) Rendimiento Académico y el puntaje total en la Prueba de Admisión: $r=0.201$ y c) Rendimiento Académico y la Subprueba de Aptitud Académica: $r=0.112$.

2.4. A nivel de las carreras o EAP las correlaciones más altas las encontramos en a) Física: entre Rendimiento Académico y el puntaje total en la Prueba de Admisión: $r=0.398$; entre Rendimiento Académico y la Subprueba de Conocimientos: $r=0.353$; b) Lingüística: entre Rendimiento Académico y la Subprueba de conocimientos: $r=0.347$; entre Rendimiento Académico y el puntaje total en la Prueba de Admisión: $r=0.335$.

2.5. A nivel de EAP, las correlaciones más bajas, e incluso negativas, entre Rendimiento Académico y la Subprueba de Aptitud Académica las hallamos en:

a) Obstetricia: entre Rendimiento Académico y la Subprueba de Aptitud Académica: $\mathrm{r}=-0.264$ : entre Rendimiento Académico y el puntaje total en la Prueba de Admisión: $r=-0.230$; entre Rendimiento Académico y la Subprueba de Conocimientos: $\mathrm{r}--0.108$.

b) Ingeniería de Sistemas: entre Rendimiento Académico y el puntaje total en la Prueba de Admisión: $\mathrm{r}=-0.092$; entre Rendimiento Académico y la Subprueba 
de Conocimientos: $\mathrm{r}=-0.083$; y entre Rendimiento Académico y la Subprueba de Aptitud Académica: $r=-0.083$.

c) Psicología: entre Rendimiento Académico y la Subprueba de Conocimientos: $\mathrm{r}=$ 0.086; entre Rendimiento Académico y la Subprueba de Aptitud Académica: $\mathrm{r}=$ 0.091; y entre Rendimiento Académico y el puntaje total en la Prueba de Admisión: $\mathrm{r}=0.104$.

3. COMPARACIÓN DE LAS CORRELACIONES ENTRE EL RENDIMIENTO ACADÉMICO Y LA PRUEBA DE PROCESAMIENTO DE LA INFORMACIÓN; Y ENTRE EL RENDIMIENTO ACADÉMICO Y LA PRUEBA DE ADMISIÓN

3.1. En la muestra total, la Prueba de Procesamiento de la Información presenta una correlación más alta con el Rendimiento Académico que la correlación entre Rendimiento Académico y la Prueba de Admisión: $\mathrm{r}=0.243$ y $\mathrm{r}=$ 0.201 ,respectivamente, ambas significativas al 0.01 , bilateral.

3.2. En la muestra total, la subprueba de conocimientos presenta una correlación con el Rendimiento Académico que supera a la correlación entre Rendimiento Académico y el puntaje total en la Prueba de Admisión de la que aquella forma parte: $r=0.242$ y $\mathrm{r}=0.201$, ambas significativas al 0.01 , bilateral. Dichas correlaciones son de magnitud similar a la correlación entre Rendimiento Académico y la Prueba de Procesamiento de la Información: $r=0.242$ y $r=0.243$, respectivamente.

3.3. A nivel de las EAP, las correlaciones entre Rendimiento Académico y las Pruebas de Procesamiento de la Información y de Admisión son semejantes en: a) Lingüística: Correlaciones de monto apreciable que implican una validez predictiva importante; $b$ ) en Ingeniería de Sistemas: las correlaciones son similares y bastantes bajas; c) en Administración las correlaciones son diametralmente opuestas: mientras que entre Rendimiento Académico y puntaje total en la Prueba de Admisión es negativa $(r=-0.089)$, entre Rendimiento Académico y el puntaje total en la Prueba de Procesamiento de la Información es positiva y de una magnitud apreciable $(r=0.286)$.

3.4. Las correlaciones son acentuadamente más altas entre Rendimiento Académico y la Subprueba de Pensamiento Estratégico: r $=0.319$, significativas al 0.01, bilateral, y entre Rendimiento Académico y el puntaje total en la Prueba de Procesamiento de la Información: $r=0.286$, significativa al 0.05 , bilateral. Tales correlaciones contrastan fuertemente con la correlación negativa entre Rendimiento Académico y la Prueba de Admisión: $\mathrm{r}=-0.289$.

Análogamente, en Obstetricia el contraste es marcado a favor de la Prueba de Procesamiento de la Información. En efecto, se presentan correlaciones negativas entre el Rendimiento Académico y la Prueba de Admisión y sus subpruebas, mientras que las correlaciones entre Rendimiento Académico y la Prueba de Procesamiento de la Información y sus subpruebas son positivas.

En Farmacia y Bioquímica ocurre lo contrario. Las correlaciones entre Rendimiento Académico y la Prueba y Subpruebas de Procesamiento de la Información son negativas o muy bajas, mientras que las correlaciones entre Rendimiento Académico y la Prueba y Subpruebas de Admisión son positivas. 
En Psicología todas las correlaciones son positivas, sin embargo, la correlación entre Rendimiento Académico y la Prueba y Subpruebas de Procesamiento de la Información superan apreciablemente a las correlaciones entre Rendimiento Académico y la Prueba y Subpruebas de Admisión.

\section{CORRELACIONES ENTRE LAS PRUEBAS Y SUBPRUEBAS DE PROCESAMIENTO DE LA INFORMACIÓN Y LAS PRUEBAS Y SUBPRUEBAS DE ADMISIÓN}

En el cuadro $\mathrm{N}^{\circ} 3$, se destacan las correlaciones entre el puntaje total en la Prueba de Procesamiento de la Información y: a) el puntaje total en la Prueba de Admisión: $r=$ 0.218 ; b) la Subprueba de Aptitud Académica: $r=0.203$; y c) la Subprueba de Conocimientos: $r=0.193$. En los tres casos las correlaciones son significativas al 0.01 , bilateral y su magnitud, que no es alta, pero tampoco es desdeñable, sugieren la conjetura de que tienen algo de común respecto a lo que miden.

Cuadro $\mathrm{N}^{\circ}$ 1. Muestra de estudio.

\begin{tabular}{|l|l|}
\hline \multicolumn{1}{|c|}{ ESCUELA ACADÉMICO PROFESIONAL } & N \\
\hline Lingüística & 25 \\
Psicología & 55 \\
Obstetricia & 56 \\
Farmacia & 63 \\
Física & 34 \\
Ingeniería de Sistemas & 66 \\
Química & 17 \\
Administración & 53 \\
Total & 369 \\
\hline
\end{tabular}

Cuadro $\mathbf{N}^{\circ}$ 2. Medias aritméticas y desviaciones estándar de los puntajes en la prueba de procesamiento de la información (total y subpruebas) por carreras y en la muestra total.

\begin{tabular}{|l|c|c|c|c|c|c|c|}
\hline \multirow{2}{*}{ EAP } & \multicolumn{3}{|c|}{ PRUEBA } & \multicolumn{2}{c|}{$\begin{array}{c}\text { SUBPRUEBA I } \\
\text { (selección argumentativa) }\end{array}$} & \multicolumn{2}{c|}{$\begin{array}{c}\text { SUBPRUEBA II } \\
\text { (pensamiento estratégico) }\end{array}$} \\
\cline { 2 - 7 } & N & $\begin{array}{c}\text { Media } \\
\text { aritmética }\end{array}$ & $\begin{array}{c}\text { Desviación } \\
\text { estándar }\end{array}$ & $\begin{array}{c}\text { Media } \\
\text { aritmética }\end{array}$ & $\begin{array}{c}\text { Desviación } \\
\text { estándar }\end{array}$ & $\begin{array}{c}\text { Media } \\
\text { aritmética }\end{array}$ & $\begin{array}{c}\text { Desviación } \\
\text { estándar }\end{array}$ \\
\hline Administración & 71 & 7.01 & 1.769 & 3.89 & 0.994 & 3.13 & 1.434 \\
Farmacia y bioquímica & 77 & 6.43 & 1.261 & 3.00 & 0.811 & 3.43 & 0.992 \\
Física & 40 & 4.60 & 1.945 & 1.95 & 1.300 & 2.65 & 1.312 \\
Ingeniería de sistemas & 82 & 5.65 & 1.636 & 2.57 & 1.133 & 3.07 & 1.109 \\
Lingüística & 71 & 5.85 & 1.818 & 2.79 & 1.041 & 3.06 & 1.132 \\
Obstetricia & 65 & 6.42 & 1.357 & 2.85 & 0.870 & 3.57 & 1.045 \\
Psicología & 72 & 6.31 & 1.805 & 2.79 & 0.855 & 3.51 & 1.444 \\
Química & 24 & 5.63 & 1.498 & 2.75 & 1.294 & 2.88 & 0.797 \\
Muestra total & 502 & 6.10 & 1.748 & 2.82 & 1.108 & 3.16 & 1.38 \\
\hline
\end{tabular}


Cuadro $\mathbf{N}^{\circ}$ 3. Coeficientes de correlación entre el rendimiento académico y los puntajes en la prueba de admisión y sus subpruebas, y entre el rendimiento académico y la prueba de procesamiento de la información, y sus subpruebas, en la muestra total y en las submuestras por carreras.

\begin{tabular}{|c|c|c|c|c|c|c|}
\hline $\begin{array}{l}\text { Rendimiento } \\
\text { académico } \\
\text { vs. }\end{array}$ & $\begin{array}{l}\text { Puntaje en } \\
\text { la prueba } \\
\text { de } \\
\text { admisión }\end{array}$ & $\begin{array}{l}\text { Puntaje en la } \\
\text { subprueba de } \\
\text { aptitud } \\
\text { académica }\end{array}$ & $\begin{array}{l}\text { Puntaje en la } \\
\text { subprueba de } \\
\text { conocimientos }\end{array}$ & $\begin{array}{c}\text { Puntaje en la } \\
\text { prueba de } \\
\text { procesamiento } \\
\text { de la } \\
\text { información }\end{array}$ & $\begin{array}{l}\text { Puntaje en la } \\
\text { subprueba de } \\
\text { selección } \\
\text { argumentativa }\end{array}$ & $\begin{array}{c}\text { Puntaje en la } \\
\text { subprueba de } \\
\text { pensamiento } \\
\text { estratégico }\end{array}$ \\
\hline Muestra total & $\begin{array}{c}* * \\
0.201\end{array}$ & $\begin{array}{c}* \\
0.112\end{array}$ & $\begin{array}{c}* * \\
0.242\end{array}$ & $\begin{array}{c}* * * \\
0.243\end{array}$ & $\begin{array}{c}* * \\
0.176\end{array}$ & $\begin{array}{c}* * \\
0.188\end{array}$ \\
\hline Lingüística & 0.335 & 0.292 & 0.347 & $\begin{array}{c}* * \\
0.224 \\
\end{array}$ & $\begin{array}{c}* \\
0.128\end{array}$ & $\begin{array}{c}* \\
0.243\end{array}$ \\
\hline Psicología & 0.104 & 0.091 & 0.086 & 0.178 & 0.114 & 0.155 \\
\hline Obstetricia & -0.230 & $\begin{array}{c}* * \\
-0.264 \\
\end{array}$ & -0.108 & 0.134 & 0.049 & 0.133 \\
\hline Farmacia & 0.151 & 0.092 & 0.174 & -0.084 & -0.163 & 0.026 \\
\hline Física & $\begin{array}{c}* \\
0.398 \\
\end{array}$ & 0.289 & $\begin{array}{c}* \\
0.353 \\
\end{array}$ & -0.028 & -0.111 & 0.068 \\
\hline $\begin{array}{c}\text { Ingeniería de } \\
\text { Sistemas }\end{array}$ & -0.092 & -0.078 & -0.083 & -0.005 & 0.023 & -0.031 \\
\hline Química & 0.127 & 0.075 & 0.141 & -0.004 & -0.163 & 0.258 \\
\hline Administración & -0.089 & & & $\begin{array}{c}* \\
0.286\end{array}$ & 0.050 & $\begin{array}{c}* * \\
0.319\end{array}$ \\
\hline
\end{tabular}

**: Correlación significativa a nivel del 0.01 (bilateral)

*: Correlación significativa a nivel del 0.05 (bilateral)

Cuadro $N^{0} 4$. Intercorrelaciones entre los puntajes en la prueba de procesamiento de la información (total y subpruebas) y los puntajes en la prueba de admisión (total y sbpruebas).

\begin{tabular}{|c|c|c|c|c|c|c|}
\hline & $\begin{array}{c}\text { Puntaje } \\
\text { total en la } \\
\text { prueba de } \\
\text { admisión }\end{array}$ & $\begin{array}{c}\text { Puntaje en la } \\
\text { subprueba de } \\
\text { aptitud } \\
\text { académica }\end{array}$ & $\begin{array}{l}\text { Puntaje en la } \\
\text { subprueba de } \\
\text { conocimientos }\end{array}$ & $\begin{array}{l}\text { Puntaje total en } \\
\text { la prueba de } \\
\text { procesamiento de } \\
\text { la información }\end{array}$ & $\begin{array}{l}\text { Puntaje en la } \\
\text { subprueba de } \\
\text { selección } \\
\text { argumentativa }\end{array}$ & $\begin{array}{c}\text { Puntaje en la } \\
\text { subprueba de } \\
\text { pensamiento } \\
\text { estratégico }\end{array}$ \\
\hline $\begin{array}{l}\text { Puntaje total en } \\
\text { la prueba de } \\
\text { admisión }\end{array}$ & & $\begin{array}{c}0.886 \\
* *\end{array}$ & $\begin{array}{c}0.915 \\
* *\end{array}$ & $\begin{array}{c}0.218 \\
* *\end{array}$ & $\begin{array}{c}0.156 \\
* *\end{array}$ & $\begin{array}{c}0.170 \\
* *\end{array}$ \\
\hline $\begin{array}{l}\text { Puntaje en la } \\
\text { subprueba de } \\
\text { aptitud } \\
\text { académica }\end{array}$ & & & $\begin{array}{c}0.625 \\
* *\end{array}$ & $\begin{array}{c}0.203 \\
* *\end{array}$ & $\begin{array}{c}0.136 \\
* *\end{array}$ & $\begin{array}{c}0.168 \\
* *\end{array}$ \\
\hline $\begin{array}{l}\text { Puntaje en la } \\
\text { subprueba de } \\
\text { conocimientos }\end{array}$ & & & & $\begin{array}{c}0.193 \\
* *\end{array}$ & $\begin{array}{c}0.145 \\
* *\end{array}$ & $\begin{array}{c}0.145 \\
* *\end{array}$ \\
\hline $\begin{array}{l}\text { Puntaje total en } \\
\text { la prueba de } \\
\text { procesamiento de } \\
\text { la información }\end{array}$ & & & & & $\begin{array}{c}0.726 \\
* *\end{array}$ & $\begin{array}{c}0.774 \\
* *\end{array}$ \\
\hline $\begin{array}{l}\text { Puntaje en la } \\
\text { subprueba de } \\
\text { selección } \\
\text { argumentativa }\end{array}$ & & & & & & $\begin{array}{c}0.127 \\
*\end{array}$ \\
\hline $\begin{array}{c}\text { Puntaje en la } \\
\text { subprueba de } \\
\text { pensamiento } \\
\text { estratégico }\end{array}$ & & & & & & \\
\hline
\end{tabular}

** : Correlación significativa al nivel del 0.01 (bilateral)

* : Correlación significativa al nivel del 0.05 (bilateral) 


\section{CONCLUSIONES}

1. Se comprueba la existencia de una marcada heterogeneidad en el conjunto de las correlaciones entre Rendimiento Académico por una parte, y, por la otra, la Prueba y Subpruebas de Procesamiento de la Información y la Prueba y Subprueba de Admisión 2003.

Los datos con los que se cuentan no son suficientes para bosquejar una hipótesis explicativa al respecto. Sin embargo, por lo hallado aquí y en otros estudios afines, cabe conjeturar que para tal finalidad explicativa se requeriría desagregar las variables como sigue: en vez de Aptitud Académica: Aptitud Verbal y Aptitud Matemática; y en vez de Conocimientos: Conocimientos-Ciencias y Conocimientos-Letras o, más exactamente: no Ciencias, esto es, el complemento de Conocimientos-Ciencias.

2. La Prueba de Procesamiento de la Información correlaciona más alto con Rendimiento Académico en las áreas de carreras de Humanidades y las que por su contenido se acercan a ellas. En cambio, correlaciona más bajo en las áreas de carreras de Ciencias e Ingenierías.

3. La Subprueba de Conocimientos de la Prueba de Admisión correlaciona algo más alto con Rendimiento Académico que la subprueba de Aptitud Académica y que la prueba total.

4. Hay indicios para suponer que la Prueba de Selección Argumentativa, por el tipo de tarea que demanda su resolución (leer atenta y analíticamente 31 párrafos para decidir qué agrupaciones de ítemes interrelacionados por su significado, responden a las preguntas que constituyen los enunciados de los ítemes), sólo funciona adecuadamente cuando se logra un alto grado de motivación en los sujetos. Si no se cumple dicha condición, decae abruptamente su eficacia.

Tal hallazgo plantea la conveniencia de utilizar dicha subprueba, tal cual, sólo con fines diagnósticos específicos, esto es, cuando la atención, que en este caso corre pareja con la motivación, es el aspecto que más importa.

Lo anterior implica la necesidad de introducir algunas modificaciones en la estructura y características de la referida subprueba para adecuarla a los propósitos primigeneamente planteados. 